\title{
Clima institucional: una revisión bibliográfica
}

\author{
Mg. Lidia Lucila Aguirre Trejo \\ lidi.lidi2808@gmail.com \\ https://orcid.org/0000-0002-5275-9743 \\ Universidad César Vallejo \\ Chimbote- Perú
}

\section{RESUMEN}

El presente trabajo reporta una revisión del concepto clima institucional con vista a profundizar el estudio, conocer los aspectos importantes para mantener y propiciar el clima institucional en las organizaciones educativas, de tal manera que el objetivo se orientó a analizar y describir los fundamentos conceptuales del clima institucional, para ello se realizó el análisis y comparación por dimensiones para evidenciar puntos de congruencia entre los autores. La investigación se caracteriza por ser descriptiva, la técnica fue la revisión de literatura de siete autores, se asume un diseño no experimental. En cuanto a las conclusiones; se realizó a base del análisis de las dimensiones como: El clima organizacional es producto de las buenas relaciones entre sus miembros, del mismo modo, es necesario propiciar la motivación, el trabajo en equipo y el desarrollo de habilidades sociales en los participantes con la finalidad propiciar cualidades para poder gerenciar o conducir a base de talentos innatos de liderazgo, de comunicación con las personas y de establecer la interacción social entre todos.

Palabras clave: clima; institucional; liderazgo; trabajo; equipo 


\title{
Institutional climate: a literature review
}

\begin{abstract}
The present work reports a review of the institutional climate concept with a view to deepening the study, knowing the important aspects to maintain and promote the institutional climate in educational organizations, in such a way that the objective was oriented to analyze and describe the conceptual foundations of climate institutional, for this, the analysis and comparison by dimensions was carried out to show points of congruence between the authors. The research is characterized by being descriptive, the technique was the literature review of seven authors, a non-experimental design is assumed. As for the conclusions; It was carried out based on the analysis of dimensions such as: The organizational climate is the product of good relationships between its members, in the same way, it is necessary to promote motivation, teamwork and the development of social skills in the participants in order to promote qualities to be able to manage or lead based on innate leadership talents, communication with people and establishing social interaction among all.
\end{abstract}

Keywords: climate, institutional, leadership, work, team

Artículo recibido: 30 noviembre. 2021 Aceptado para publicación: 29 diciembre 2021

Correspondencia: lidi.lidi2808@gmail.com Conflictos de Interés: Ninguna que declarar 


\section{INTRODUCCIÓN}

A nivel internacional, el COVID-19 ha perjudicado los diversos sectores sociales como en lo económico, en la salud de las personas, la educación en todos sus niveles y campos, de tal manera que ha determinado cambio en el desarrollo de las actividades de las personas, en su comportamiento, en la manera de trabajar entre otros aspectos. Abordando el tema desde el ámbito educativo, en nuestro contexto ante la situación misma que pasa por el problema de la pandemia del COVID-19, las clases fueron suspendidas hasta un nuevo aviso; esto también ha generado acciones o maneras de trabajo y de relaciones personales entre los docentes, estudiantes, directivos y padres de familia; de tal manera que ante la situación presentada el clima institucional ha dejado de evidenciarse en las instituciones educativas de manera presencial, ahora también se percibe de una manera virtual.

Pacheco, Rojas, Niebles, Hernández, y Durán (2020), La relación interpersonales es necesario para el desarrollo o el avance social, fundamentándose en una buena comunicación y comprensión de la realidad, la armonía de igual manera contribuye a un buen entendimiento entre las personas; en tal sentido contribuye a un buen clima organizacional.

En ese sentido, Pacheco,et.al (2020), consideró que el clima institucional es uno de los aspectos fundamentales para que la gestión marche bien en las instituciones, específicamente en las instituciones educativas, en donde se orienta a considerar y mejorar las estrategias para el funcionamiento de las organizaciones, asimismo se necesita ciertos factores para dar consolidación a la importancia del clima institucional como la motivación, las relaciones humanas, el desarrollo de las habilidades blandas y por ende el compromiso institucional.

Parra, Margel y Rocha, Grace y Durán, Sonia (2021), consideran "la importancia de las líneas normativas y métodos para el buen desempeño alineados a las tendencias de la organización, además de aplicación de método de supervisión inflexible e impreciso" (p.10). Cabe considerar que se refiere a un marco normativo que también ayuda a contribuir a un buen clima institucional; es decir los actores deben de asumir responsabilidades y compromiso en las instituciones y en sus áreas de trabajo.

En consecuencia, es necesario dentro de la organización hacer prevalecer las normas, acatar los dispositivos; es decir asumir con responsabilidad los acuerdos, considerando 
para ello asumir mejores estrategias y métodos de realizar un trabajo más organizados y cohesionado entre los participantes.

Para Durán y Parra (2016), es necesario que en las organizaciones de hoy en día deben de considerar como fundamental el recurso humano; en donde es necesario alcanzar los objetivos planteados mediante la comunicación, el desarrollo del liderazgo y el buen trato horizontal entre los participantes (p.225).

En la actualidad encontramos en las organizaciones diferentes modelos o formas como se dirige a sus trabajadores, depende del tipo de gestión y liderazgo, este conjunto de variables determina el clima institucional u organizacional; es decir depende de la forma o manera de cómo se gestiona las organizaciones (Fonseca Montoya et al., 2019).

De esta manera, el clima institucional se acentúa más en el campo educativo, en donde refiere al comportamiento de los actores tanto a los docentes, estudiantes y directivos, en tal sentido que: "La gestión del clima institucional constituye un elemento importante en el desempeño del personal docente en la excelencia de su trabajo, y por tanto, en la motivación y aprendizaje de sus estudiantes" (More y Morey,2021, p.21).

Todo se orienta en busca de la excelencia, de tal manera que el sentido del clima institucional no solamente tiene que ver con asumir los objetivos desde un punto normativo por parte de los actores educativos, sino que va más allá como es el compromiso en las diversas actividades académicas, administrativas y pedagógicas.

En nuestro contexto, urge la necesidad de conocer la importancia del clima institucional, como los docentes, directivos, administrativos y estudiantes se involucran en las actividades, saber el desarrollo de sus habilidades inter e intra personales, Parra, et.al (2020), consideró que para lograr el clima institucional depende también del liderazgo dentro de la organización, la autoridad debe ser influyente, empático, sea quien comprenda a los demás actores. Iglesias, Torres \& Mora (2020), en sus aportes considera que en las organizaciones siempre se debe de realizar las evaluaciones o diagnóstico en el aspecto emocional, personal y grupal, desarrollar técnicas y estrategias que ayude a identificar comportamientos o actitudes desfavorables de la persona para poder asumir decisiones o solución al problema de los actores.

De acuerdo a lo antes expuesto en el artículo se pretende determinar ¿De qué manera se percibe el clima institucional en las instituciones educativas del distrito del Santa?; y 
como objetivo general se ha considerado: Establecer un análisis de cómo se percibe el clima institucional en las instituciones educativas del distrito del Santa.

\section{ESTRATEGIAS METODOLÓGICAS O MATERIALES Y MÉTODOS}

El estudio de la temática permitió indagar y analizar sobre la incidencia del clima institucional como un factor de importancia en el desarrollo de la organización educativa y de esta manera conocer como contribuye a la identidad, a la misión y visión; es decir como contribuye a la filosofía institucional como características o prestancia de una buena institución.

El método empleado se sustenta en un nivel descriptivo Según Hernández, Fernández y Baptista (2010) la investigación descriptiva "busca especificar propiedades, características y rasgos importantes de cualquier fenómeno que analice" (p.80). De la misma manera, se ha realizado consulta bibliográfica- periodo 2021 con respecto a la variable de estudio, se considerará también el aporte y opiniones de directores, sub directores y docentes involucrados en el desarrollo del clima institucional. Asimismo, la revisión de documentos normativos en donde reflejan el aseguramiento y la responsabilidad de contribuir al buen clima institucional en las organizaciones educativas de la provincia.

\section{RESULTADOS Y DISCUSIÓN}

A continuación, son respecto a la variable de estudio que es el clima institucional, presentamos mediante el cuadro de comparación a siete autores quienes dan sustento al tema; entre ello: More, Renzo, A., \& More (2020); Espíritu, A., \& Castro, J. (2020); Castillo, E., Medina, M., Bernardi, J., Reyes, C., \& Ayala, C. (2019); Angulo, C.(2020); Pariente, I(2020) y Quispe. (2020), en los cuales en sus investigaciones se relacionan con la variable de clima institucional, por lo cual describimos los resultados de acuerdo a sus apreciaciones (ver tabla 1).

En cuanto el clima institucional, Arenas (2020) y Chata (2019), coinciden como clima institucional está referido las buenas relaciones humanas en una institución en donde evidencie relaciones laborales, relaciones humanas, entre los trabajadores y el empleador; es decir, como lo define Cena, Blaz, \& Castro (2021). Que una institución es: “un organismo público o privado para ejercer una función o labor política, social, cultural o científica" (p.67). En ese sentido, el clima institucional se construye, se socializa, fortalece con las buenas relaciones humanas entre sus miembros o participantes. 
Con respecto a la dimensión de liderazgo, son abordados por More, Renzo, A., \& More (2020), el liderazgo es la cualidad que tiene un directivo para influenciar en los demás, es necesario manejar las relaciones humanas de tal manera que contribuya a la solidez del clima institucional, en ese sentido tenemos a Espíritu \& Castro (2020) en donde aborda que el clima institucional es el producto de la buenas relaciones entre sus miembros, de tal manera que genera confianza, seguridad y responsabilidad en el desarrollo de las cosas. Además, Acosta (2020), establece que el clima institucional es importante para las organizaciones porque así genera competitividad y calidad en todos sus procesos.

En cuanto a la dimensión de la motivación; Espíritu, A., \& Castro, J.(2020); Castillo; Medina; Bernardi.; Reyes, \& Ayala, C.(2019); relacionan a la variable clima institucional con la motivación, en el cual señala que es un componente importante en el clima institucional y a la vez se considera un factor importante para estimular a los miembros de la organización y poder lograr los objetivos; la motivación genera dinamismo entre los miembros de la organización. Finalmente, en la apreciación de De la Cruz \& Salazar (2020), como de Espíritu \& Castro (2020), enfatiza que el clima institucional, enfatiza la importancia del hombre en función de su trabajo y por la participación en un sistema social, esto demuestra la interacción entre un ámbito laboral en el cual debe de compartir lazos sociales, laborales y de actividades en común.

El trabajo en equipo es otro de los atributos en los cuales refleja las apreciaciones de los autores, así tenemos a Enciso (2020), el trabajo en equipo en las instituciones resulta vital, se debe de propiciar el trabajo en equipo porque contribuye a que todas las actividades propuestas conlleve a los resultados, Espíritu \& Castro (2020), el trabajo en equipo se debe de propiciar y debe penetrar en la cultura organizacional; es decir formar parte de una filosofía de trabajo en las organizaciones, de esta manera genera compromiso en asumir las actividades de la institución; en definitiva un buen clima de trabajo en equipo, genera y favorece el aprendizaje organizacional y el compromiso de equipos de trabajo. En ese sentido, Angulo (2020), refiere el desarrollo de las habilidades blandas; es decir es necesario desarrollar en los miembros de la organización habilidades socio afectivas; es decir personas que sean emprendedores, activos, que asuman compromiso y reto y a la vez con cualidad valorativa del valor humano, que sea empático, que valore el trabajo de los demás y contribuya al desarrollo de las actividades. Castillo; Medina; Reyes \& Ayala (2020), para conducir al clima organizacional y contribuir a la buena gestión, es necesario 
desarrollar habilidades para la vida, lógicamente que las habilidades blandas son importantes por caracterizarse por ser más asertivo o empático, pero esto va más allá; sostiene que es necesario desarrollar en las personas habilidades sociales; es decir habilidades interpersonales (comunicación asertiva, confianza, cooperación y empatía), habilidades interpersonales (habilidades para el trabajo en equipo, socialización) y otra habilidad relacionado a la habilidad emocional (manejo de emociones, el estrés, el carácter) de esta manera, el manejo de estas habilidades se necesita desarrollar en todos los sujetos de la organización.

Al respecto de las habilidades gerenciales, Fernández (2018), Merino (2021) y Molina (2018), está referido a la capacidad, a la manera de cómo llevar en adelante la organización, esta habilidad se va perfeccionando mediante la experiencia laboral con la que cuente el gerente; es decir va adquiriendo esta experticia a lo largo de la vida profesional. Sagredo (2019), coincide con Pariente (2020), en donde señala la importancia de desarrollar habilidades y además de propiciar estrategias para manejar grupos humanos, para distribuir o asignar funciones y para tomar decisiones en los momentos más cruciales.

Otro elemento que es impórtate para contribuir al clima institucional es la comunicación, la comunicación debe de ser horizontal, debe ser fluida entre sus miembros, alturada, de la conversación, de la lluvia de ideas y de las expresiones ante los demás sale grandes aportes, Angulo (2020), Enciso (2020), refiere que la comunicación importante desde lo más básico que debe brindar la persona como saludar y escuchar con atención, son habilidades esenciales para el desarrollo de una comunicación horizontal, esto evita malas interpretaciones y ayuda a desarrollar empatía y vínculos que fortalecerán en gran medida una armonía en el trabajo participativo.

En cuanto a la motivación, el docente experimenta emociones que interactúan con sus pensamientos, lo cual lo pone en acción; al respecto, según Valenzuela, Muñoz, y Montoya (2018) la motivación es un factor importante en el comportamiento de la persona ya que asegura su persistencia e identidad y calidad de compromiso institucional, por ello es importante reconocer y retribuir el aporte individual a cada profesor de parte de toda organización en mérito a su eficiencia. 


\section{CONCLUSIÓN O CONSIDERACIONES FINALES}

El clima institucional es el reflejo del trabajo en equipo y el desarrollo de un conjunto de habilidades interpersonales, intrapersonales y emocionales que contribuye en el logro de los planes u objetivos planteados en la organización.

Propiciar el trabajo en equipo, de tal manera el grupo de personas comparte ideas, destrezas similares, capacidad y decisiones en conjunto; es importante porque ayuda a maximizar las fortalezas de cada trabajador y miembro del equipo, de la misma manera crea sinergia, fomenta la responsabilidad y la capacidad de respuesta al cambio, finalmente; impulsa el trabajo multidisciplinario en las organizaciones.

Propiciar las habilidades gerenciales, de tal manera que proporcionan instrumentos indispensables para la gestión; es decir las estrategias y métodos para tomar las decisiones, elegir de manera racional las diferentes alternativas u opciones a base de las propuestas dadas.

La motivación es otro factor interesante, impulsa a los miembros de la organización a lograr la productividad, mejora la calidad humana en las personas, una persona motivada se siente segura, en confianza, encuentra satisfacción con lo que hace y aún más cuan la persona es valorada por su empresa; contribuye al buen clima organizacional.

\section{LISTA DE REFERENCIAS}

Abreu, S. A. (2017). La ètica en la investigación educativa. Instituto Internacional de Investigación y Desarrollo Tecnológico Educativo INDTEC, C.A. Revista Scientific, 2(4), 338-350. http://scielo.sld.cu/pdf/rus/v12n4/2218-3620-rus-1204-333.pdf

Acosta, L.(2020). Mejoramiento del clima institucional a través de estrategias de gestión en los docentes de la IE José Lorenzo Cornejo Acosta de Arequipa. VÉRITAS Vol. $21 \mathrm{~N}^{\circ} 2$ (2020) 21-23

Arenas, I. S. P. (2020). Mejoramiento del clima institucional a través de estrategias de gestión en los docentes de la IE José Lorenzo Cornejo Acosta de Arequipa. Veritas, 21(2), 21-23.

Angulo,W. (2020). Habilidades gerenciales en el clima institucional de un Ministerio Público de Lima, 2019.https://repositorio.ucv.edu.pe/bitstream/handle/20.500.12692/41054/Angul o_CW.pdf? sequence $=1 \&$ isAllowed=y. 
Castillo, E., Medina, M., Bernardi, J., Reyes, C., \& Ayala, C. (2019). Leadership and organizational climate in workers of health facilities of a micronetwork in Perú. Revista Cubana de Salud Pública, 45(2), 1 13. Recuperado de https://scielosp.org/pdf/rcsp/2019.v45n2/e1351/ es

Chata, R. (2019). Estilo de liderazgo y clima organizacional en instituciones educativas de gestión pública del distrito de Coata-Puno. Revista de Investigaciones de La

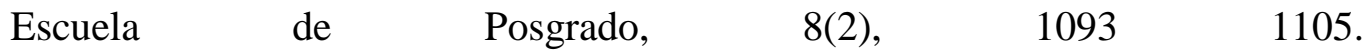
https://doi.org/http://dx.doi.org/10.26788/riepg.2 019.2.129

Cenas-Chacón, F. Y., Blaz-Fernández, F. E., \& Castro-Mendocilla, W. E. (2021). Clima institucional y desempeño docente en instituciones educativas del distrito La Esperanza-UGEL N 02-2020. Polo del Conocimiento, 6(5), 964-979.

De la Cruz, E. E. D., \& Salazar, T. D. R. M. (2020). Relación entre clima institucional y desempeño docente en las instituciones educativas de la ugel $\mathrm{n}^{\circ}$ 03-Trujillo2017. Perfiles de las Ciencias Sociales, 8(15).

.Durán, S. E., y Parra, M. A. (2016). Condiciones laborales y calidad de vida en el trabajo: Un desafío para la gestión de talento humano en Venezuela. En R. Prieto y R. De La Hoz (Eds.), Estrategias innovadoras: Acontando los retos de la economía global (pp. 437-459). Universidad Autónoma del Estado de Hidalgo y Universidad Autónoma de Tlaxcala.

Espíritu Martínez, Antony Paul, \& Castro Llaja, Lindomira. (2020). Clima organizacional y gestión de servicios de salud por enfermería del Hospital Daniel Alcides Carrión. Conrado, 16(76), 157-168. Epub 02 de octubre de 2020. Recuperado en 12 de noviembre de 2021, de http://scielo.sld.cu/scielo.php?script=sci_arttext\&pid=S1990$\underline{86442020000500157 \& \operatorname{lng}=\text { es\&tlng=es. }}$

Enciso (2020). Clima institucional y desempeño de docentes en instituciones educativas de la Asociación Educativa Adventista de Puno. NAWPARISUN - Revista de Investigación Científica, Vol. 2, Num. 2, (Enero - Marzo, 2020).

Fernández, M. (2018). Habilidades gerenciales en el clima institucional de la Corte Suprema de Justicia del Perú, 2018. (Tesis Doctoral) Universidad César Vallejo. https://repositorio.ucv.edu.pe/bitstream/handle/20.500.12692/26739/zapata_rw.p df? sequence $=1 \&$ isAllowed $=\mathrm{y}$ 
Fonseca Montoya, S., Lucio Chávez, E. D., \& Sánchez Gálvez, S. (2019). Una mirada a tres lustros de educación continua y postgraduada en la Universidad de Guayaquil (1990-2016). El caso de la Facultad de Filosofía, Letras y Ciencias de la

Educación. Conrado, 15(67), 14-23. http://scielo.sld.cu/scielo.php?script=sci_arttext\&pid=S1990$\underline{86442019000200014 \& \text { lang }=p t}$

Hernández, R. Fernández C. y Baptista P. (2010). Metodología de la investigación organizacional (4ta. ed.). México: McGraw-Hill. https://www.uv.mx/personal/cbustamante/files/2011/06/Metodologia-de-laInvestigaci\%C3\%83\%C2\%B3n_Sampieri.pdf

Iglesias Armenteros, Annia Lourdes, \& Torres Esperón, Julia Maricela, \& Mora Pérez, Yuliett (2020). Estudios de clima organizacional: revisión integrativa. MediSur, 18 (6), 1189-1197. [Fecha de Consulta 5 de Noviembre de 2021]. ISSN:. Disponible en: https://www.redalyc.org/articulo.oa?id=180065571015

Merino Lloclla, Y. (2021). Clima institucional percibido en la Institución Educativa Santa Rosa de la provincia de Sullana antes y durante el confinamiento 2020. http://purl.org/pe-repo/ocde/ford\#5.03.00.

Molina, Y. (2018). Estilo de liderazgo gerencial en el desempeño laboral en la Dirección Regional Agraria de Huancavelica- 2018. (Tesis de título). Universidad Nacional de Huancavelica, Perú. Disponible en: repositorio.unh.edu.pe/bitstream/../TP\%20\%20UNH\%20ADMIN.\%200064.pdf

More Espinoza, Renzo Augusto, \& Morey Guevara, Marco Osias. (2021). Gestión del clima institucional y desempeño del personal docente de la Institución Educativa N 7044 San Martín de Porres, Chorrillos. Dilemas contemporáneos: educación, política y valores, 8(spe2), 00006. Epub 21 de abril de 2021.https://doi.org/10.46377/dilemas.v8i.2588

Parra, Margel y Rocha, Grace y Durán, Sonia (2021). Liderazgo como prospectiva del clima organizacional en el sector hotelero. Revista de Ciencias Sociales (Ve), 27 (2), 217-227. [Fecha de Consulta 1 de Noviembre de 2021]. ISSN: 1315-9518. Disponible en: https://www.redalyc.org/articulo.oa? id=28066593013 
Pacheco, C. M., Rojas, C. P., Niebles, W. A., Hernández, H. G., y Durán, S. E. (2020). Estrategias motivacionales para caracterizar el clima organizacional en el sector salud. Revista Espacios, 41(29), 1-17. https://doi.org/10.46377/dilemas.v8i.2588

Pariente, I. (2020). Mejoramiento del clima institucional a través de estrategias de gestión en los docentes de la IE José Lorenzo Cornejo Acosta de Arequipa. VÉRITAS Vol. 21 N² (2020) 21-23. file:///C:/Users/USUARIO/Downloads/273-1-626-210-20201105.pdf.

Quispe Fernandez, E. (2020). Clima institucional en el desempeño docente en las II. EE. de la Red Educativa de Ccarhuayo, UGEL Quispicanchis-Cusco.

Ricse, K. (2018). Clima institucional y satisfacción laboral en los docentes de la institución educativa Santa Rosa de Trujillo. Año 2017. (Tesis de maestria). Universidad César Vallejo.

Sagredo Lillo, E. J. (2019). Relaciones entre gestión organizacional y percepción del compromiso, motivación y satisfacción de estudiantes y docentes de Centros educativos de adultos de la Región del Biobío Chile [Tesis doctoral para optar al grado de Doctor en Psicología de la Comunicación y Cambio Universitat Autónoma de Barcelona]

Valenzuela, J., Muñoz, C., \& Montoya, A. (2018). Estrategias motivacionales efectivas en profesores en formación. Educação e Pesquisa, 44(0), 120. https://doi.org/10.1590/s1678- 4634201844179652. 